Palavras chave:

Perfilamento a laser

Análise de regressão

Manejo florestal de precisão

Amazônia

Histórico:

Recebido 20/03/2013

Aceito 29/04/2014

Keywords:

Laser profiling

Regression analysis

Precision forestry

Amazon

Correspondência: evandro.figueiredo@embrapa.br

DOI: I 0. I590/0 I04776020|42004|693
Evandro Orfanó Figueiredo', Marcus Vinicio Neves d'Oliveira', Philip Martin Fearnside², Daniel de Almeida Papa'

\section{MODELOS PARA ESTIMATIVA DE VOLUME DE ÁRVORES INDIVIDUAIS PELA MORFOMETRIA DA COPA OBTIDA COM LIDAR}

RESUMO: A estimativa volumétrica, a partir do escaneamento digital de florestas por meio do uso do LIDAR, potencializa o emprego de técnicas de manejo de precisão no planejamento da exploração nas florestas tropicais. A utilização dessa tecnologia de sensoriamento remoto permite a incorporação de variáveis da morfometria de copa, ainda pouco empregadas e menos conhecidas em decorrência da dificuldade de coleta em campo. $O$ objeto deste estudo foi construir equações capazes de estimar o volume do fuste de árvores individuais dominantes e codominantes, a partir da morfometria da copa obtida por meio do LIDAR aerotransportado, considerando duas situações de inventário florestal: a) com a coleta do DAP, conjuntamente com as variáveis morfométrica da copa obtidas pelo LIDAR e b) apenas com os dados de morfometria de copa. Para seleção dos modelos foram considerados: a matriz de correlação das variáveis preditoras e a combinação das variáveis que geraram os melhores resultados estatísticos pelos critérios $\mathrm{S}_{y \mathrm{x}}, \mathrm{S}_{\mathrm{yx}}(\%)$ e Press, e que foram homocedásticos e com disposição dos resíduos normais e independentes. Para as melhores equações foram realizadas análise de influência. Os resultados estatísticos do ajuste dos modelos para as duas situações permitiram selecionar equações com e sem DAP, com resultados $\mathrm{R}_{\mathrm{ai}}^{2}$ (\%) de a) 92,92 e b) 79,44 ; $\mathrm{S}_{\mathrm{yx}}$ (\%) de a) 16,73 e b) 27,47 ; e, critério de Press de a) $20 \mathrm{I}, 15 \mathrm{~m}^{6}$ e b) $537,47 \mathrm{~m}^{6}$, respectivamente. Por meio das variáveis morfométricas, foi possível desenvolver equações capazes de estimar com precisão o volume do fuste de árvores dominantes e codominantes em florestas tropicais.

\section{MODELS TO ESTIMATE VOLUME OF INDIVIDUAL TREES BY MORPHOMETRY OF CROWNS OBTAINED WITH LIDAR}

ABSTRACT: The volumetric estimate from digital scanning of the forests through the use of LIDAR increases the precision of forest management techniques in planning tropical forest logging operations. The use of this remote detection technology allows the incorporation of crown morphometric variables which are still little known and little used due to the difficulty of collecting field data for volume equations. The objective of this study was to build equations capable of estimating the stem volume of dominant and codominant individual trees from the crown's morphometry obtained by airborne LIDAR, considering two forest inventory situations: a) with the collection of diameter at breast height $(\mathrm{DBH})$, and crown morphometric variables obtained from LIDAR data and b) using only the crown morphometry variables. For the selection of models the factors considered were: the correlation matrix of predictor variables and the combination of variables that generates the best results by statistical criteria $S_{y x}, S_{y x}(\%)$ and Press ${ }_{p}$, and that were homoscedastic and had a normal and independent distribution of errors. The influence analysis was performed for the best equations. The results for the statistical fit of the equations to the two situations allowed the selection of models with and without $\mathrm{DBH}$, with $\mathrm{R}_{\text {ai. }}^{2} \%$ ) values of a) 92.92 and b) $79.44, \mathrm{~S}_{\mathrm{yx}}(\%)$ values of a) 16.73 and b) 27.47 , and, Press criterion values of a) $201.15 \mathrm{~m}^{6}$ and b) $537.47 \mathrm{~m}^{6}$, respectively. Through morphometric variables it was possible to develop equations capable of accurately estimating the stem volume of dominant and codominant trees in tropical forests.

' Embrapa Acre - Rio Branco, Acre, Brasil

${ }^{2}$ Instituto Nacional de Pesquisas da Amazônia - Manaus, AM, Brasil 


\section{INTRODUÇÃO}

Os planos de manejo florestal na Amazônia Brasileira são licenciados com base em critérios que buscam, principalmente, garantir a qualidade dos dados dendrométricos referentes ao volume do fuste, número de indivíduos e resíduos florestais para produção de energia. Apesar dos avanços na qualidade das informações florestais com emprego de técnicas de precisão com o uso do receptor GPS e geoprocessamento incorporados ao planejamento da exploração florestal (FIGUEIREDO et al., 2007), os custos e a morosidade do inventário censitário para florestas tropicais, continuam sendo um problema de base tecnológica a ser resolvido.

No meio florestal, a expectativa da adoção do perfilamento por meio do LIDAR (Light Detection And Ranging) aerotransportado é que os inventários proporcionarão uma combinação desejada de baixo custo, precisão, qualidade e confiabilidade.

A estimativa volumétrica, a partir do escaneamento digital de florestas, potencializará o uso de técnicas de precisão no planejamento da exploração florestal, tornando essas estimativas um importante instrumento de consolidação do manejo. Com isso, esperase que o planejamento reflita melhor a realidade de campo, a exemplo dos resultados de estimativas de volume, biomassa, área basal e contagem de árvores obtidos por Gonçalves-Seco et al. (20II) e loki et al. (20I0).

Quando se trata de estimativas biométricas por árvore individual com o LIDAR aerotransportado, necessariamente serão agregadas aos modelos volumétricos, as variáveis da morfometria da copa ainda pouco empregadas e menos conhecidas (POPESCU et al., 2003). Os resultados de pesquisa com a morfometria de copa de árvores individuais pelo perfilamento com LIDAR aerotransportado, ainda são escassos e limitamse a florestas plantas ou naturais no hemisfério norte, conforme Andersen et al. (20I I), Bottai et al. (20l3) e Holmgren et al. (2003). Em razão das dificuldades de coleta em campo, as variáveis morfométricas eram preteridas, e agora ganham destaque pela forma rápida e segura com que os dados são obtidos por meio do perfilamento da floresta.

A morfometria de copa é o estudo da matemática das formas em uma mesma população de árvores. Atualmente, as formas e dimensões das árvores e sua modificação com o tempo adquirem nova importância, dada a possibilidade de ajustamento de modelos estatísticos de estimativa volumétrica, concorrência e de crescimento (HASENAUER et al., 1995).
Objetivou-se, neste estudo, desenvolver equações para floresta tropical na Amazônia capazes de estimar o volume do fuste de árvores individuais dominantes e codominantes, a partir da morfometria da copa obtida por meio do perfilamento florestal com LIDAR aerotransportado, considerando duas situações de levantamento: a) com a coleta em campo do diâmetro à altura do peito (DAP), conjuntamente com variáveis morfométrica da copa e b) apenas com os dados de morfometria de copa.

\section{MATERIAL E MÉTODOS}

\section{Localização e caracterização da área de estudo}

Os estudos foram desenvolvidos em área de plano de manejo florestal da Floresta Estadual do Antimary - FEA, localizada nos municípios de Bujari e Sena Madureira (AC), numa área de 315 hectares.

O clima é classificado como Awi (Köppen) com precipitação anual média de $2.000 \mathrm{~mm}$, temperatura média de $25^{\circ} \mathrm{C}$ e uma estação seca entre os meses de junho e setembro. A vegetação e composta por três fisionomias florestais principais: florestas densas, florestas abertas e florestas abertas com bambus (tabocas). A área possui uma topografia suave ondulada com elevação máxima em torno dos 300 metros. Os solos predominantes são latossolos amarelos distróficos (ACRE, 2000).

\section{Formação da base de dados}

A base de dados foi organizada em quatro categorias, sendo: I) base de dados do inventário censitário pelas técnicas preconizadas pelo Modelo Digital de Exploração Florestal (FIGUEIREDO et al., 2007): foi realizada a identificação e localização geográfica das árvores de interesse comercial e obtenção dos pontos barométricos calibrados por meio de GPS de alta sensibilidade. Foram coletadas exsicatas das espécies que compõem a amostra e depositadas no Herbário da Universidade Federal do Acre (UFACPZ); 2) arquivos de nuvens de pontos de reflexão do LIDAR de 315 hectares de floresta; 3) dimensão e arquitetura das copas das árvores da amostra, obtidas pelo processamento dos dados do LIDAR e verificações de campo; e, 4) dados de cubagem rigorosa pelo método de Smalian.

A Tabela I, apresenta-se a estrutura da amostragem proporcional estratificada por classe diamétrica das árvores de interesse que foram cortadas e cubadas, visando a compor a base de dados para o 
ajuste dos modelos volumétricos. Ressalta-se, aqui, que a amostra seguiu o mesmo padrão de distribuição diamétrica da floresta inventariada, de maneira a atender os critérios legais de exploração e condicionantes ambientais da Licença de Operação do Instituto de Meio Ambiente do Acre - IMAC, N530/2008 e Certificação SmartWood no: SW-FM/COC-1670.

\section{Levantamento florestal com o LIDAR aerotransportado e pré-processamento}

O perfilamento pelo LIDAR ocorreu entre 29 de maio a 3 de junho de 2010, utilizando-se o sistema Optech ALTM 3100 EA acoplado a um avião bimotor Piper Seneca II, modelo Neiva/Embraer 8I0C. O voo foi realizado à velocidade média de $210 \mathrm{~km} \cdot \mathrm{h}^{-1}$, numa altura de $300 \mathrm{~m}$, diâmetro do feixe de $20 \mathrm{~cm}$, divergência do feixe de $0,3 \mathrm{mrad}$, ângulo de varredura de $15^{\circ}$, frequência de varredura de $58,7 \mathrm{~Hz}$ e intensidade média de 43,03 pontos por metro quadrado (OLIVEIRA et al., 20I2).

Os dados de reflexão do LIDAR, referentes à área florestal, foram estruturados inicialmente em 315 arquivos de nuvens de pontos. Posteriormente, foram montados mosaicos com área média de 25 hectares e cerca de II milhões de pulsos. Os arquivos foram processados em base tridimensional em software específico (Quick Terrain Modeler).

A etapa seguinte foi a localização das 2.09। árvores dominantes e codominantes de interesse, inventariadas em campo com a apropriação de uma coordenada geográfica com GPS de alta sensibilidade. As coordenadas apropriadas em campo pelo inventário receberam a transformação geocêntrica nos parâmetros $X, Y, Z$ visando à localização exata das árvores de interesse no arquivo de pontos do LIDAR.

Os dados de nuvens de pontos receberam tratamento conjunto com a aplicação de filtros de cores, considerando a altura e a textura da superfície alvo, além da combinação de um valor do modelo digital de elevação e a textura da superfície do alvo. Posteriormente, foi aplicado um controle de reflexão denominado de Voxel Autosize, em que pontos projetados na superfície são processados em tamanhos diferentes, com base em sua posição em relação ao plano de visualização, ou seja, pontos que estão próximos ao plano de visualização ficam maiores, enquanto que os pontos que estão longe do plano de visualização ficam menores (APPLIED IMAGERY, 20I0). Esse processo facilita o desenho do contorno de árvores dominantes e codominates, e, consequentemente, $\circ$ processo de isolamento das árvores.

O isolamento dos pontos do LIDAR das árvores da amostra foi feito por meio da confecção de um polígono tridimensional na região do entorno da copa. Essa região foi recortada da nuvem de pontos total. Essa operação serviu para garantir que todos os pontos fossem selecionados numa determinada região $X Y Z$, incluindo a árvore de interesse e a vegetação sob a sua copa.

A árvore foi isolada da vegetação do subbosque seguindo o mesmo procedimento de confecção de polígonos e recortes sucessivos, porém agora em duas dimensões (Figura I). Independentemente da espécie florestal, as variáveis explicativas das árvores individuais, constituiram $\circ$ conjunto de dados para $\circ$ desenvolvimento de modelos volumétricos globais.

\section{Variáveis independentes obtidas da morfometria de copa}

Para constituir a base de dados das variáveis independentes dos modelos volumétricos foram consideradas duas situações de levantamento florestal. A primeira considerou a coleta do DAP em campo e todas as variáveis dos índices morfométricos da copa obtidas pelo perfilamento a laser, e a segunda apenas os dados morfométricos da copa obtidas pelo LIDAR, sem considerar o emprego do DAP.

TABELA I Base de dados da amostragem proporcional estratificada por classe diamétrica da população de árvores dominantes e codominantes cubadas para o ajuste dos modelos volumétricos.

TABLE I Proportional sampling data base stratified by diameter class of the dominant and codominant trees with volumes measured for the fitting of volumetric models.

\begin{tabular}{|c|c|c|c|c|c|}
\hline $\begin{array}{c}\text { Classes } \\
\text { diamétricas }\end{array}$ & $\begin{array}{l}\text { Intervalo das } \\
\text { classes }(\mathrm{cm})\end{array}$ & $\begin{array}{c}\mathrm{N}^{\circ} \text { indivíduos da } \\
\text { população inventariada }\end{array}$ & (\%) & $\begin{array}{l}\mathrm{N}^{\circ} \text { indivíduos para o ajuste } \\
\text { dos modelos de volume }\end{array}$ & (\%) \\
\hline I & $45 \vdash 74,9$ & 1294 & 61,88 & 88 & 60,27 \\
\hline II & $75 \vdash 104,9$ & 570 & 27,26 & 41 & 28,08 \\
\hline III & $105 \vdash 134,9$ & 174 & 8,32 & 12 & 8,22 \\
\hline IV & $135 \vdash 164,9$ & 53 & 2,53 & 5 & 3,42 \\
\hline Total & & 2091 & 100,00 & 146 & 100,00 \\
\hline
\end{tabular}


(a)

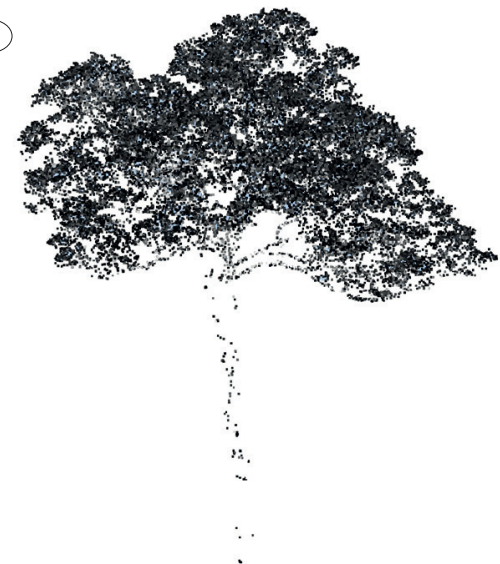

(b)

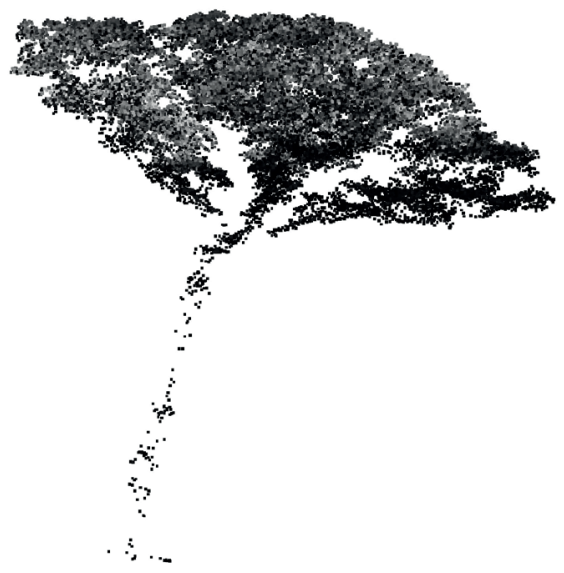

(c)

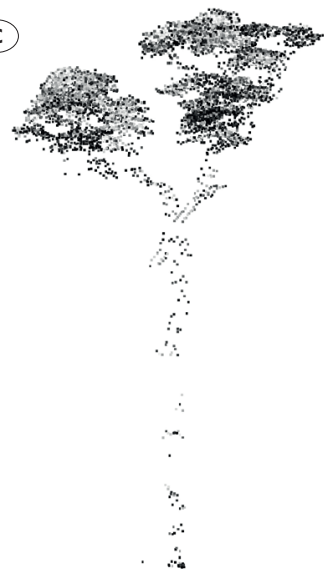

FIGURA I Árvores isoladas da vegetação de sub-bosque com dados do LIDAR: a) Dipteryx odorata Wild (cumaru ferro), b) Ceiba pentandra (L.) Gaertn. (samaúma), c) Apuleia leiocarpa (Vogel) J.F. Macbr. (cumaru cetim), Floresta Estadual do Antimary - FEA.

FIGURE I Trees isolated from the understory vegetation using LIDAR data: a) Dipteryx odorata Wild (cumaru ferro), b) Ceiba pentandra (L.) Gaertn. (samaúma), c) Apuleia leiocarpa (Vogel) J.F. Macbr. (cumaru cetim), Antimary State Forest - FEA.

Assim, foi feita uma adaptação das variáveis morfométricas da árvore (Figura 2), propostas por Burger (1939), para a construção dos modelos para estimar o volume, sendo: comprimento de copa (Cc), comprimento da galhada $(\mathrm{Cg})$, diâmetro médio da copa (DC), altura total da árvore $(\mathrm{Ht})$, altura de inserção da copa $(\mathrm{Hic})$, proporção de copa $(\mathrm{PC}=\mathrm{Cc} / \mathrm{Ht})$, grau de esbeltez $(\mathrm{GE}=\mathrm{Ht} / \mathrm{DAP})$, índice de saliência $(\mathrm{IS}=\mathrm{DC} /$ $\mathrm{DAP})$, índice de abrangência $(\mathrm{IA}=\mathrm{DC} / \mathrm{Ht})$, formal de copa $(F C=D C / C c)$, índice de espaço vital $(\mathrm{IEV}=(\mathrm{DC} /$ $\mathrm{DAP})^{2}$ ), volume de copa pelo sólido de rotação que melhor modela a copa (VCop), área de projeção de copa (APC) e manto de copa (MC), além da cota de elevação do terreno no ponto de localização da árvore (Hsolo). As variáveis $\mathrm{Cc}, \mathrm{Cg}, \mathrm{DC}, \mathrm{Ht}$ e as procedentes destas foram obtidas pelo processamento do dados do LIDAR e verificadas em campo. As variáveis VCop, APC e MC foram obtidas na forma digital pelo LIDAR, sem a averiguação de campo, em decorrência da dificuldade de mensurar essas informações na floresta.

\section{Estimativa do volume do fuste}

Os volumes da cubagem rigorosa das 146 árvores dominantes e codominantes constituem os dados da variável dependente dos modelos de regressão e os índices morfométricos da copa (com e sem DAP) que compõem as variáveis independentes.

Os dados tabelados foram submetidos a uma análise de correlação para determinar intensidade da relação linear entre a variável dependente $e$ as preditoras, e, assim, apontar, inicialmente, as variáveis mais significativas para a construção dos modelos.

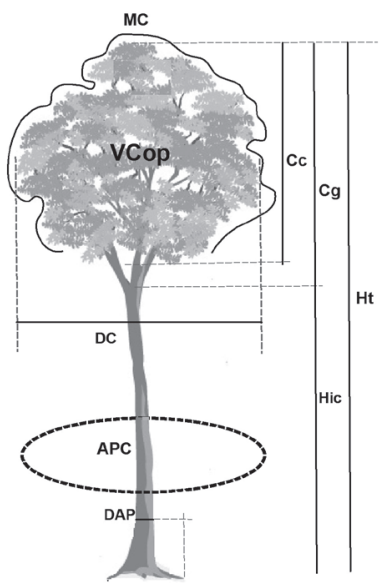

$\mathrm{Cc}=$ Comprimento de copa $(\mathrm{m})$

$\mathrm{Cg}=$ Comprimento de galhada $(\mathrm{m})$

$\mathrm{DC}=$ Diâmetro médio da copa $(\mathrm{m})$

$\mathrm{Ht}=$ Altura total da árvore $(\mathrm{m})$

$\mathrm{DAP}=$ Diâmetro à altura do peito a $1,3 \mathrm{~m} /$ coletado em campo $(\mathrm{cm})$

$\mathrm{Hic}=$ Altura de inserção da copa $(\mathrm{m})$

$\mathrm{APC}=$ Área de projeção de copa $\left(\mathrm{m}^{2}\right)$

Vcop = Volume pelo sólido de rotação que melhor modela a copa $\left(\mathrm{m}^{3}\right)$ $\mathrm{MC}=$ Manto de copa (área superficial do sólido de rotação em $\mathrm{e}^{2}$ )

FIGURA 2 Variáveis morfométricas da árvore empregando dados do LIDAR e DAP. Adaptado de Burger ( 1939).

FIGURE 2 Tree morphometric variables using LIDAR data and DBH. Adapted from Burger (1939).

As variáveis independentes com coeficiente de correlação inferior a $-0,65$ e superior a 0,65 , foram submetidas a uma rotina de seleção de todos os modelos possíveis (RYAN, 20II) que ponderam a inclusão de 2 a 6 parâmetros $\left(\beta_{\text {is }}\right)$, sem multicolinariedade, com distribuição normal dos resíduos, independentes e homocedásticos. Para isso, foram realizadas as seguintes 
estatísticas: matriz de multicolinariedade, distribuição do erro padronizado, teste de Durbin-Watson (DW) e o F-máximo de Hartley (STATGRAPHICS, 2006; STATISTICAL ANALYSIS SYSTEM INSTITUTE - SAS INSTITUTE, 1990).

Para cada um dos modelos indicados pela rotina de seleção (RYAN, 20II) foi obtida uma equação de volume e examinada a significância estatística de cada variável adicionada por meio do teste de Fischer (teste F). As variáveis com nível de significância $<0$, I5 foram retiradas do polinômio visando a sua simplificação. 0 estabelecimento de um parâmetro 0,15 ou 0,20 se faz necessário para evitar que durante o processo de desenvolvimento, remova-se e inclua a mesma variável explicativa em sucessivos passos (STATGRAPHIS, 2006).

Para cada equação de volume ajustada, foi realizada uma análise de influência das observações. Foram consideradas observações influentes aquelas que provocam diferenças substanciais nos valores calculados da estatística na presença e ausência da observação. Para a avaliação dos impactos das possíveis observações influentes para modelos lineares, foram adotados os seguintes medidores de atipicidade: elementos da diagonal principal da matriz H, DFFITS e distância de Cook's.

A seleção do melhor modelo foi baseada na análise gráfica dos resíduos em porcentagem, no erro padrão absoluto e percentual $\left(\mathrm{S}_{\mathrm{yx}}\right.$ e $\left.\mathrm{S}_{\mathrm{yx}} \%\right)$, no critério Press e no coeficiente de determinação ajustado $\left(R_{\text {aj }}^{2} \%\right)$.

$O$ critério Press ${ }_{p}$ é uma medida que avalia se a equação ajustada pode predizer os valores observados $Y_{i}$. Assim, cada valor ajustado $\left(\hat{Y}_{i}\right)$ é alcançado eliminandose o i-ésimo caso do conjunto de dados e ajusta-se uma nova função de regressão para o modelo em questão com as (n-I) observações restantes. Em cada nova equação, para obter o valor predito $\left(\hat{Y}_{\mathrm{i}(\mathrm{i})}\right)$ da i-ésima observação, estima-se o erro de predição Press ${ }_{p}$ do i-ésimo caso $\left(Y_{i}\right.$ $\left.\hat{Y}_{i(i)}\right)$. Assim, o critério Press consiste na soma de todos esses erros de predição quadráticos e funciona como um instrumento de validação da equação, onde os melhores ajustes geram os menores valores de Press ${ }_{\mathrm{p}}$ (SAS, 1990; STATGRAPHIS, 2006).

\section{RESULTADOS E DISCUSSÃO}

\section{Volume do fuste obtidos pelos índices morfométrico considerando o DAP}

Os resultados da matriz de correlação apontaram que as variáveis DAP, área de projeção de copa (APC), diâmetro médio da copa (DC), índice de abrangência (IA), manto da copa (MC) e volume da copa
(VCop) apresentaram correlação positiva superior a 0,65 e o grau de esbeltez (GE) e a elevação do terreno (Hsolo) com correlação negativa inferior a $-0,65$. Portanto, para a construção dos modelos de volume, as demais variáveis morfométricas foram descartadas em decorrência de sua correlação não significativa.

Os melhores resultados de correlação foram observados para o DAP e o manto da copa (MC), este último com correlação positiva com o volume do fuste superior a 0,80. Apesar dos valores de manto da copa (MC) serem obtidos somente pelo processamento digital da nuvem de pontos do LIDAR, essa variável se mostrou bastante promissora como preditora do volume, juntamente com a área de projeção da copa (APC) e o volume da copa (VCop).

A rotina de combinação das variáveis independentes possibilitou selecionar dez conjuntos de variáveis para construção das equações de volume (Tabela 2).

A obtenção de estimativas de volume por árvores individuais é o princípio para um planejamento operacional no manejo florestal, e o uso de variáveis morfométricas da copa tendem a melhorar a precisão dessas estimativas, conforme resultados observados na equação I. Nenhum dos modelos que usam exclusivamente as variáveis independentes, tradicionalmente empregadas (DAP, Ht) obtiveram resultados melhores que modelos que também aplicam variáveis oriundas dos índices de morfometria de copa. Resultados inferiores com equações de simples (DAP) e de dupla entrada (DAP, Ht) na Amazônia, também foram obtidos por Barros e Silva Júnior (2009) e Silva (2007).

A equação I, obtida pelo processo de seleção (STATGRAPHIS, 2006), alcançou os melhores resultados estatísticos (Tabela 2), cujo gráfico de resíduo percentual não apresenta uma tendência marcante de super ou subestimativa ao longo da amplitude dos volumes (Figura 3a), sendo recomendada para uso. Na Figura 3 b, quando se observam os volumes reais e estimados, verificam-se que os valores estimados apresentam uma boa distribuição e proximidade com os valores reais, mostrando a adequabilidade da equação.

\section{Volume do fuste obtido pelos índices morfométrico sem o DAP}

Os resultados da matriz de correlação são os mesmos descritos anteriormente, porém, em decorrência da retirada do DAP das variáveis independentes, não foi considerado a inclusão do grau de esbeltez (GE). 
TABELA 2 Equações ajustadas para estimar o volume do fuste de árvores dominantes e codominantes com uso do DAP e dos índices de morfometria da copa, com seus respectivos coeficientes de determinação ajustado, erro padrão (absoluto e percentual) e critério Press.

TABLE 2 Fitted equations to estimate stem volume of dominant and codominant trees from DBH and crown morphometry variables, with their respective adjusted coefficients of determination, standard errors (absolute and percentage), and Press criterion values.

\begin{tabular}{|c|c|c|c|c|c|}
\hline$N^{\circ}$ & Equação & $\begin{array}{l}\mathrm{R}_{\text {aj. }}^{2} \\
(\%)\end{array}$ & $\begin{array}{c}S_{y x} \\
\left(m^{3}\right)\end{array}$ & $\begin{array}{l}S_{y x} \\
(\%)\end{array}$ & $\begin{array}{c}\text { Press }_{\mathrm{p}} \\
\left(\mathrm{m}^{6}\right)\end{array}$ \\
\hline $\mathrm{I}^{*}$ & $V F=-16,0666+0,244099 \cdot D A P+0,109968 \cdot G E-0,00826033 \cdot A P C+0,0000153755 \cdot A P C^{2}$ & 92,92 & $\pm 1,218$ & 16,73 & 201,16 \\
\hline 2 & $V F=1,6275+0,00 I I 387 \cdot D A P^{2}+0,00000327065 \cdot A P C^{2}-4,573 I 4 \cdot I A+0,000476786 \cdot V C o p$ & 88,28 & $\pm 1,380$ & 19,64 & 272,34 \\
\hline 3 & $V F=-15,5 \mid 19+0,231758 \cdot D A P+0,0895972 \cdot G E+0,00000677426 \cdot A^{2} C^{2}-0,0000540946 \cdot V C o p$ & 90,12 & $\pm 1,400$ & 19,69 & 277,13 \\
\hline 4 & $V F=-4,55759+0,00135084 \cdot \mathrm{DAP}^{2}+0,0587816 \cdot \mathrm{GE}+0,0000023229 \cdot \mathrm{APC}^{2}+0,000226159 \cdot \mathrm{VCop}$ & 88,23 & $\pm 1,379$ & 19,73 & 267,41 \\
\hline 5 & $V F=I, 57443+0,00116249 \cdot D^{2} P^{2}+0,0000077315 \cdot A^{2} C^{2}-3,95997 \cdot I A$ & 88,13 & $\pm 1,423$ & 20,01 & 289,29 \\
\hline 6 & $V F=-5,\left|5278+0,00139448 \cdot D_{A P}^{2}+0,068\right| 84 \mid \cdot G E+0,00000446226 \cdot A^{2} C^{2}$ & 87,84 & $\pm 1,440$ & 20,25 & 294,36 \\
\hline 7 & $V F=-0,12709+0,0011201 \cdot D A P^{2}+0,00000680945 \cdot A^{2} C^{2}$ & 87,59 & $\pm 1,440$ & 20,52 & 286,78 \\
\hline 8 & $V F=-0,0559 \mid 8 I+0,00 I I I 787 \cdot \mathrm{DAP}^{2}+0,0000045223 \mathrm{I} \cdot \mathrm{MC}^{2}$ & 87,42 & $\pm 1,451$ & 20,67 & 291,82 \\
\hline 9 & $V F=-6,67487+0,173926 \cdot D A P+0,0000083663 \cdot A P C^{2}$ & 87,48 & $\pm 1,450$ & 20,71 & 280,52 \\
\hline 10 & $V F=-0,737966+0,00116706 \cdot D^{2} P^{2}+0,00369934 \cdot A P C$ & 86,28 & $\pm 1,473$ & 21,26 & 298,60 \\
\hline
\end{tabular}

$\mathrm{VF}=$ volume do fuste $\left(\mathrm{m}^{3}\right)$; DAP = diâmetro à altura do peito $(\mathrm{cm}) ; \mathrm{GE}=$ grau de esbeltez; APC = área de projeção da copa $\left(\mathrm{m}^{2}\right) ; \mathrm{IA}=$ índice de abrangência; VCop = volume pelo sólido de rotação que melhor modela a copa $\left(\mathrm{m}^{3}\right) ; \mathrm{MC}=$ manto de copa (área superficial do sólido de rotação em $\left.\mathrm{m}^{2}\right) ; \mathrm{R}_{\mathrm{aj}}^{2}(\%)$ = coeficiente de determinação ajustado em percentagem; $\mathrm{S}_{\mathrm{yx}}\left(\mathrm{m}^{3}\right)$ = erro padrão absoluto; $\mathrm{S}_{\mathrm{yx}}(\%)=$ erro padrão percentual; Press $_{\mathrm{p}}=$ critério de Press $\left(\mathrm{m}^{6}\right)$; e, * equação recomendada para uso.
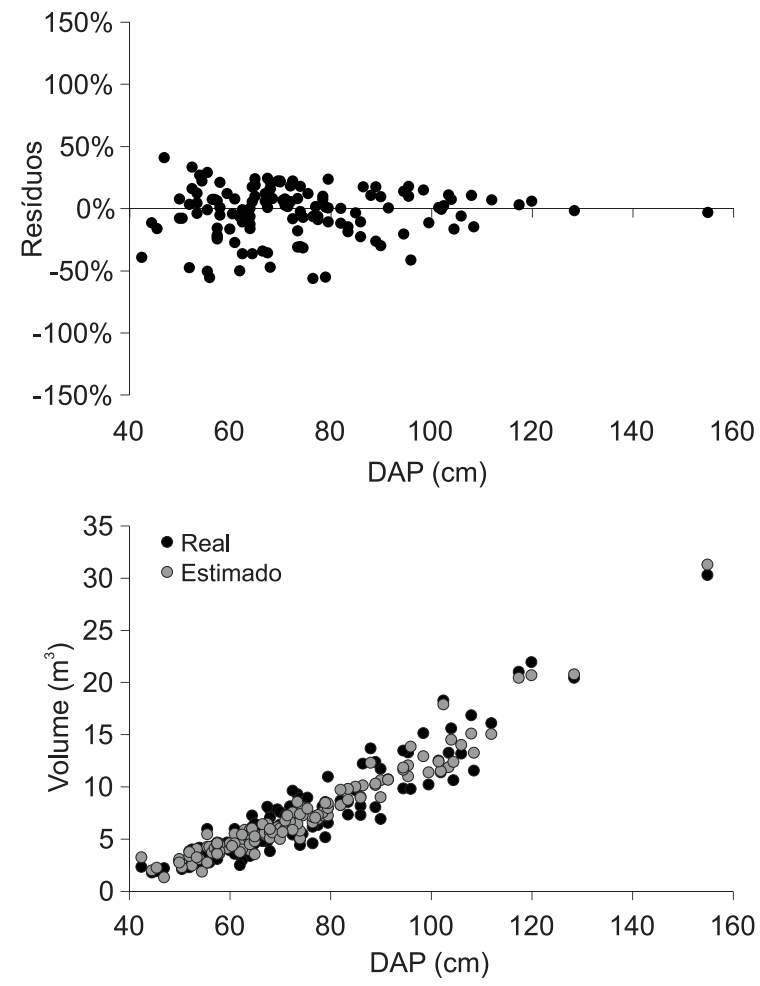

FIGURA 3 (a) Distribuição dos resíduos percentuais e (b) comportamento dos volumes estimados em relação aos volumes reais da equação recomendada.

FIGURE 3 (a) Distribution of residuals in percentage and (b) estimated vs. observed volumes for the recommended equation.
$\mathrm{Na}$ Tabela 3, são apresentadas as estatísticas para as nove equações selecionadas pela combinação das variáveis com maior correlação com o volume do fuste, desconsiderando o DAP. Essas equações apresentam resultados para $\mathrm{R}^{2}{ }_{\mathrm{aj} .}(\%)$, variando de 72,68 a 79,44 e erro padrão percentual $\mathrm{S}_{y x}(\%)$ entre 27,47 a 30,84 . As equações com o uso exclusivamente de variáveis de copa apresentaram resultados estatísticos inferiores aos obtidos pelas equações selecionadas com o DAP. Entretanto, quando se considera a equação recomendada (3), os resultados são similares ou até mesmo melhores que os alcançados pelas equações de simples entrada para Amazônia, elaboradas por Barros e Silva Júnior (2009), Rolim et al. (2006) e Silva (2007).

loki et al. (2010) e Roberts et al. (2005) obtiveram resultados similares para equações de estimativas biométricas (DAP, Ht, diâmetro de copa e volume), porém, em situações de floresta plantada e de clima temperado. Estudos semelhantes para floresta tropical são escassos e apresentam um grande campo do conhecimento ainda a ser trabalhado.

$\mathrm{Na}$ Figura 4a observa-se que no gráfico de resíduo percentual para equação recomendada (3), há uma superestimativa para o volume das árvores com DAP entre 40 a $70 \mathrm{~cm}$ e uma pequena subestimativa entre os diâmetros de 100 a $110 \mathrm{~cm}$. Isso aponta que os dados volumétricos podem ser tratados diferentemente por classe diamétrica visando a melhorar a precisão dos 
TABELA 3 Equações ajustadas para estimar o volume do fuste de árvores dominantes e codominantes, a partir dos índices de morfometria da copa, com seus respectivos coeficientes de determinação ajustado, erro padrão (absoluto e percentual) e critério Press.

TABLE 3 Fitted equations to estimate tree volume of dominant and codominant trees from the crown morphometry with their respective adjusted coefficients of determination, standard errors (absolute and percentage), and Press ${ }_{p}$ criterion values.

\begin{tabular}{|c|c|c|c|c|c|}
\hline $\mathrm{N}^{\circ}$ & Equação & $\begin{array}{l}\mathrm{R}^{2} \\
(\%)\end{array}$ & $\begin{array}{c}\mathrm{S}_{y x} \\
\left(\mathrm{~m}^{3}\right)\end{array}$ & $\begin{array}{l}S_{y x} \\
(\%)\end{array}$ & $\begin{array}{c}\text { Press } \\
\left(\mathrm{m}^{6}\right)\end{array}$ \\
\hline I & $V F=2,05704+0,014205 \cdot D C^{2}+0,0397898 \cdot$ Hsolo $-19,0759 \cdot I A+0,00000884883 \cdot M^{2}$ & 77,89 & 2,158 & 28,44 & 587,98 \\
\hline 2 & $V F=9, I 59+0,0000133954 \cdot A_{P C}^{2}+0,014548 \mid \cdot D C^{2}-18,9683 \cdot I A$ & 74,50 & 2,075 & 28,33 & 533,07 \\
\hline $3^{* *}$ & $V F=9,88824+0,0|14846| \cdot D C^{2}-20,3348 \cdot I A+0,00000879273 \cdot M C^{2}$ & 79,44 & 2,085 & 27,47 & 537,47 \\
\hline 4 & $V F=9,68207+0,0158245 \cdot D C^{2}-19,0128 \cdot 1 A+0,0000110549 \cdot M C^{2}-0,000685208 \cdot V C o p$ & 78,09 & 2,160 & 28,57 & 597,33 \\
\hline 5 & $V F=27,44|4-1,48095 \cdot D C+0,048599| \cdot D^{2}-|7,583| \cdot \mid A-639,827 \cdot M^{-1}$ & 78,02 & 2,201 & 28,60 & 607,82 \\
\hline 6 & $V F=4,98352+0,502895 \cdot D C-19,0156 \cdot I A+0,0000 I I 8297 \cdot M C^{2}$ & 74,09 & 2,144 & 28,91 & 577,30 \\
\hline 7 & $V F=0,989362+0,0000132477 \cdot \mathrm{APC}^{2}+0,0139819 \cdot \mathrm{DC}^{2}+0,0432504 \cdot$ Hsolo- I8,2355.IA & 72,68 & 2,149 & 29,07 & 573,39 \\
\hline 8 & $V F=-4,50708+0,487974 \cdot D C+0,0521928 \cdot$ Hsolo $-18,8065 \cdot I A+0,00001|688| \cdot M^{2}$ & 73,11 & 2,186 & 29,09 & 595,06 \\
\hline 9 & $V F=12,3288+366,902 \cdot A P C^{-1}+0,0142687 \cdot D C^{2}-23,0855 \cdot I A+8,8763 E-6 \cdot M C^{2}-629,726 \cdot M^{-1}$ & 76,09 & 2,361 & 30,84 & 724,80 \\
\hline
\end{tabular}

Hsolo = cota de elevação do terreno no ponto de localização da árvore $(\mathrm{m}) ; \mathrm{VF}=$ volume do fuste $\left(\mathrm{m}^{3}\right) ; \mathrm{DC}=$ diâmetro médio da copa $(\mathrm{m})$, APC = área de projeção da copa $\left(\mathrm{m}^{2}\right) ; \mathrm{IA}=$ índice de abrangência; $\mathrm{VCop}=$ volume pelo sólido de rotação que melhor modela a copa $\left(\mathrm{m}^{3}\right) ; \mathrm{MC}=$ manto de copa (área superficial do sólido de rotação em $\left.\mathrm{m}^{2}\right) ; \mathrm{R}_{\mathrm{aj}}^{2}(\%)=$ coeficiente de determinação ajustado em percentagem; $\mathrm{S}_{\mathrm{yx}}\left(\mathrm{m}^{3}\right)=$ erro padrão absoluto; $\mathrm{S}_{\mathrm{yx}}(\%)=$ erro padrão percentual; Press ${ }_{\mathrm{p}}=$ critério de Press $\left(\mathrm{m}^{6}\right)$; e ** equação recomendada para uso.
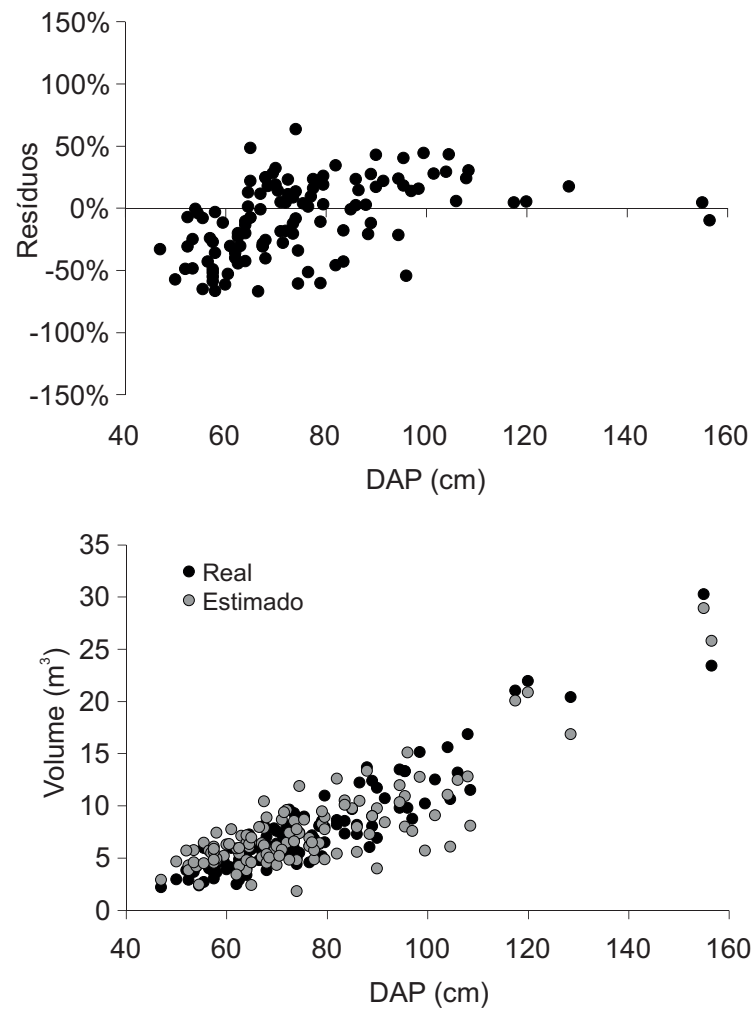

FIGURA 4 (a) Distribuição dos resíduos percentuais e (b) comportamento dos volumes estimados em relação aos volumes reais da equação recomendada (sem DAP).

FIGURE 4 (a) Distribution of residuals in percentage and (b) estimated vs. observed volumes for the recommended equation (without $\mathrm{DBH}$ ). modelos. Porém, há necessidade de ampliar a base de dados nas diferentes classes diamétricas, principalmente para as árvores de maior diâmetro. $\mathrm{Na}$ Figura 4b, o gráfico do volume real e estimado nota-se uma dispersão maior dos volumes estimados, principalmente entre os diâmetros de 90 a $110 \mathrm{~cm}$.

\section{CONCLUSÕES}

Os índices de morfometria obtidos com os dados do LIDAR: área de projeção da copa (APC), grau de esbeltez (GE), índice de abrangência (IA), manto da copa (MC), volume da copa (VCop) e diâmetro médio da copa (DC), conjuntamente, com a elevação do terreno (Hsolo) e o DAP foram as variáveis independentes mais adequadas para estimar o volume do fuste, gerando equações de boa precisão em toda a amplitude de classes de diâmetro, com destaque para equação I $(\mathrm{VF}=-16,0666+0,244099 \cdot \mathrm{DAP}+0,109968 \cdot \mathrm{GE}-$ $\left.0,00826033 \cdot \mathrm{APC}+0,0000153755 \cdot \mathrm{APC}^{2}\right)$.

As equações volumétricas que adotaram exclusivamente variáveis de copa obtiveram resultados estatísticos similares as equações de simples entrada (com DAP) em estudos dendrométricos na Amazônia. $O$ melhor ajuste foi obtido pela equação $3(V F=9,88824+$ $0,0|4846| \cdot D C^{2}-20,3348 \cdot \mid A+0,00000879273 \cdot M C^{2}$ ), a qual deve ser empregada para futuros planejamentos operacionais do manejo, quando se realizar o perfilamento florestal pelo LIDAR aerotransportado. 


\section{REFERÊNCIAS}

ACRE. Zoneamento ecológico-econômico: recursos naturais e meio ambiente. Rio Branco: SECTMA, 2000. II $6 \mathrm{p}$.

ANDERSEN, H. E.; STRUNK, J.; TEMESGEN, H.; ATWOOD, D.; WINTERBERGER, K. Using multi-level remote sensing and ground data to estimate forest biomass resources in remote regions: a case study in the boreal forests of interior Alaska. Canadian Journal of Remote Sensing, Kanata, v. 37 , n. 6 , p. 596-6II, 201 I

APPLIED IMAGERY. Quick terrain modeler: LIDAR exploitation software: user's manual. Version 7. Maryland, 2010. $232 \mathrm{p}$

BARROS, P. L. C.; SILVA JÚNIOR, A. T. Equação de volume para árvores de uma floresta tropical densa no município de Anapu, oeste do estado do Pará, Amazônia Oriental. Ciências Agrárias, Belém, n. 5I, p. II5-I26, 2009.

BOTTAI, L.; ARCIDIACO, L.; CHIESI, M.; MASELLI, F. Application of a single-tree identification algorithm to LiDAR data for the simulation of stem volume current annual increment. Journal of Applied Remote Sensing, Bellingham, v. 7, n. I, p. 73699-73699, 2013.

BURGER, H. Baumkrone und zuwachs in zwei hiebsreifen fichtenbeständen. Mitteilungen der Schweizerischen Anstalt für das Forstliche Versuchswesen, Zurich, v. 21, p. 147-176, 1939

FIGUEIREDO, E. O.; BRAZ, E. M.; OLIVEIRA, M. V. N. d'. Manejo de precisão em florestas tropicais: modelo digital de exploração florestal. Rio Branco: EMBRAPA Acre, 2007. $183 \mathrm{p}$.

GONÇALVES - SECO, L.; GONZÁLEZ - FERREIRO, E.; DIÉGUEZ - ARANDA, U. Assessing the attributes of highdensity Eucalyptus globulus stands using airborne laser scanner data. International Journal of Remote Sensing, London, v. 32, n. 24, p. 982I-984I, 201 I.

HASENAUER, H.; MOSER, M.; ECKMÜLLNER, O. Ein Programm zur Modellierung von Wachstumsreaktionen. Allgemeine Forstzeitung, Ghent, v. 4, p. 216-218, 1995.
HOLMGREN, J.; NILSSON, M.; OLSSON, H. Estimation of tree height and stem volume on plots using airborne laser scanning. Forest Science, Bethesda, v. 49, n. 3, p. 419428, 2003.

IOKI, K.; IMANISHI, J.; SASAKI, T.; MORIMOTO, Y.; KITADA, $\mathrm{K}$. Estimating stand volume in broad-leaved forest using discrete-return LiDAR: plot-based approach. Landscape and Ecological Engineering, Tokyo, v. 6, n. I, p. 29-36, 2010.

OLIVEIRA, M. V. N. d'; REUTEBUCH, S. E.; MCGAUGHEY, R. J.; ANDERSEN, H. E. Estimating forest biomass and identifying low-intensity logging areas using airborne scanning lidar in Antimary State Forest, Acre State, Western Brazilian Amazon. Remote Sensing of Environment, New York, v. I24, p. 479-49I, 2012.

POPESCU, S. C.; WYNNE, R. H.; NELSON, R. F. Measuring individual tree crown diameter with LIDAR and assessing its influence on estimating forest volume and biomass. Canadian Journal of Remote Sensing, Ontario, v. 29, n. 5, p. 564-577, 2003

ROBERTS, S. D.; DEAN, T. J.; EVANS, D. L. Estimating individual tree leaf area in loblolly pine plantations using LiDARderived measurements of height and crown dimensions. Forest Ecology and Management, Amsterdam, v. 213, n. I/3, p. 54-70, 2005.

ROLIM, S. G.; THADEU, H.; COUTO, Z.; JESUS, R. M. de; FRANÇA, J. T. Modelos volumétricos para a Floresta Nacional do Tapirapé-Aquirí, Serra dos Carajás, PA. Acta Amazonica, Manaus, v. 36, n. I, p. I07-I I4, 2006.

RYAN, T. P. Estatística moderna para engenheira. Rio de Janeiro: Elsevier, 201 I. 324 p.

STATISTICAL ANALYSIS SYSTEM INSTITUTE. SAS user's guide: statistics. Release 6.03. Cary, 1990. 584 p.

SILVA, J. L. R. Modelos volumétricos, fatores de forma e equação de afilamento para floresta de terra firme da região do rio Aru, município de Portel-Pará. 2007. 85 p. Dissertação (Mestrado em Silvicultura e Manejo Florestal) - Universidade Federal Rural da Amazônia, Belém, 2007.

STATGRAPHICS. Statgraphics Centurion XV: user's guide. New York, 2006. 299 p. 